\title{
Journal of the National Comprehensive Cancer Network
}

The Last Word

\section{Palliative Care: Who is Responsible?}

\author{
Amanda M. Rosenblum, MD, CCFP (PC), and \\ Martin R. Chasen, MBChB, MPhil (Pall Med)
}

In a time when medicine has been fairly criticized as producing too many subspecialists, a positive movement toward multidisciplinary care teams has begun. Oncology programs are no exception. Although many cancer centers have dedicated teams based on the primary cancer, individual members within these teams represent multiple disciplines. For example, at minimum, a head and neck cancer team should include a medical oncologist, radiation oncologist, surgical oncologist, nurse, dietitian, speech language pathologist, and social worker. For patients with advanced cancer, multiple randomized studies have shown that early palliative care improves patient quality of life, and in some instances quantity of life. ${ }^{1}$ Therefore, an oncology program is not complete without palliative care.

In "Outcomes From a Patient-Centered, Interprofessional, Palliative Consult Team in Oncology" published in this issue, Feldstain et al skillfully demonstrate not only the statistical but also the clinical benefits of interdisciplinary specialist palliative care in reducing symptom burden and detecting distress in patients with high intensity scores. Their team consistently used 2 validated tools at patient encounters: the Edmonton Symptom Assessment System (ESAS) and the Canadian Problem Checklist (CPC). The ESAS is a questionnaire that asks patients to rate the severity of 9 common symptoms-pain, fatigue, tiredness, nausea, appetite, shortness of breath, depression, anxiety, and wellbeing-on a visual analogue scale ranging from 0 to $10 .^{2}$ The CPC is a checklist of the most commonly reported problems, divided into the following domains of functioning: physical, emotional, social/family, spiritual, informational, and practical, although the version used by Feldstain and colleagues also included mobility. ${ }^{3}$

Similarly, the NCCN Clinical Practice Guidelines in Oncology (NCCN Guidelines) for Distress Management recommend use of the NCCN Distress Thermometer (on which patients rate their distress from $0-10$ ) and Problem List as brief initial screening tools at all clinical visits. ${ }^{4}$ Similar to the CPC, the Problem List includes the following domains: physical problems, practical problems, family problems, emotional problems, and spiritual or religious concerns. A Distress Thermometer score $\geq 4$ is considered a positive result, and should prompt further questioning and potentially a referral to the appropriate provider based on the source or sources of distress identified. Screening for distress beyond physical sources improves recognition of the impact that social determinants of health have on cancer care. Although these tools are not new to most palliative care programs, their uptake into standard oncology practice has not been universal. ${ }^{5}$

Yet, palliative care also finds itself at a critical juncture in ensuring equitable and accessible delivery. Feldstain et al note that their team has tried to expand beyond the ambulatory oncology setting, using phone calls to improve patient follow-up. However, they also mention that several different palliative care teams operate within the same geographic area, providing care in different settings such as the home or hospice.

Several models of delivery exist for palliative care. Primary palliative care refers to the basic competencies and skills all physicians (and ideally all healthcare providers) should have to address symptom management and goals-of-treatment conversations. Specialist palliative care refers to the skills for managing patients with more challenging and complex needs. ${ }^{6}$ Thus, although most patients with cancer would benefit from a supportive and palliative care approach, aimed at treating symptoms and reducing suffering, not all patients require referral to a specialist palliative care physician.

Although consensus referral criteria for specialist palliative care have been proposed for the outpatient and inpatient settings, ${ }^{7,8}$ primary palliative care must not go underrecognized or undersupported, because not only are there not enough specialist palliative care providers to meet the demand but this would also further isolate and fragment patient

doi: 10.6004/jncen.2018.7047

The ideas and viewpoints expressed in this commentary are those of the author and do not necessarily represent any policy, position, or program of NCCN. 


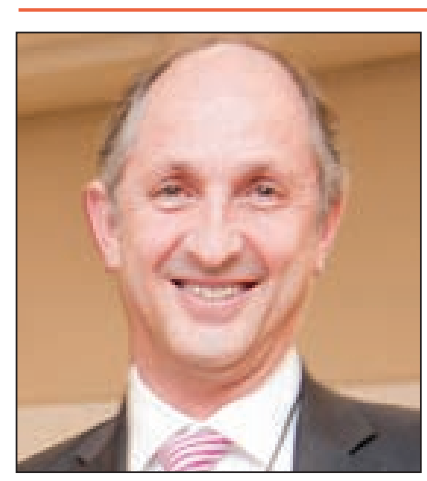

Martin R. Chasen, MBChB, MPhil (Pall Med)

Martin R. Chasen, MBChB, MPhil (Pall Med), is the Medical Director of Palliative Care at William Osler Health System in Brampton. He currently holds professorships at the Universities of Toronto, McMaster, Ottawa, and McGill. Dr. Chasen has lectured nationally and internationally on rehabilitation of patients with cancer, approaches to treatment of patients with cachexia, chemotherapy-induced nausea and vomiting, survivorship, and symptom relief. care. ${ }^{6}$ Instead, specialist palliative care physicians should act as resources for and advisers to clinicians providing primary palliative care, whether it is the primary treating oncologist or the family physician, and be reserved for managing more complex cases.

Although high intensity scores on distress screening tools may indicate a need for a specialized referral, other specialized areas, such as psychosocial oncology, have shown that many of the trigger criteria can be managed with more frequent assessment in the primary clinic first, and that patients can be referred out only when necessary. ${ }^{9}$ Feldstain et al found that low-intensity symptom scores increased at the next follow-up appointment. This finding has been previously published, and various reasons have been hypothesized, including the volatility of symptoms and the relation to underlying disease progression. ${ }^{10}$ Again, the primary treating team may be able to manage these patients, and may not necessarily need to refer them to specialized palliative care.

Promising developments have been seen in terms of incorporating palliative care competencies into medical training. For example, the Canadian Medical Association's policy reinforces the need for all physicians to have at least basic competencies in palliative care, and has requested that all Canadian faculties of medicine create a training curriculum in palliative care regardless of training level or practice setting. ${ }^{11}$ For healthcare providers already in practice, several courses aimed at teaching palliative care fundamentals are available, such as Pallium Canada's Learning Essential Approaches to Palliative and End-of-Life Care (LEAP; http://pallium.ca/leap-courses/). LEAP also includes several subspecialty courses, such as LEAP Onco for healthcare providers working in cancer care programs.

Finally, patients and their caregivers are crucial members of the cancer care team. Healthcare providers must empower them to take ownership of their health and symptoms by creating an environment that allows them to feel comfortable voicing concerns. Use of a general self-efficacy framework has previously been shown to effectively reduce depression symptoms in patients with cancer. ${ }^{12}$

Palliative care remains everyone's responsibility.

\section{References}

1. Bakitas MA, El-Jawahri A, Farquhar M, et al. The TEAM approach to improving oncology outcomes by incorporating palliative care in practice. J Oncol Pract 2017;13:557-566.

2. Bruera E, Kuehn N, Miller MJ, et al. The Edmonton Symptom Assessment System (ESAS): a simple method for the assessment of palliative care patients. J Palliat Care 1991;7:6-9.

3. Talk About What Matters to You-Putting Patients First. Alberta Health Services Website. Available at: http:// www.albertahealthservices.ca/frm-20338.pdf. Accessed October 13, 2017.

4. Holland JC, Andersen B, Breitbart WS, et al. Clinical Practice Guidelines in Oncology: Distress Management. Version 2.2013. J Natl Comp Canc Netw 2013;11:190-209. To view the most recent verson of these guidelines, visit NCCN.org.

5. Zebrack B, Kayser K, Bybee D, et al. A practice-based evaluation of distress screening protocol adherence and medical service utilization. J Natl Compr Canc Netw 2017;15:903-912.

6. Quill TE, Abernethy AP. Generalist plus specialist palliative care-creating a more sustainable model. N Engl J Med 2013;368:1173-1175.

7. Hui D, Mori M, Watanabe SM, et al. Referral criteria for outpatient specialty palliative cancer care: an international consensus. Lancet Oncol 2016;17:e552-559.

8. Weissman DE, Meier DE. Identifying patients in need of a palliative care assessment in the hospital setting: a consensus report from the Center to Advance Palliative Care. J Palliat Med 2011;14:17-23.

9. Li M, Macedo A, Crawford S, et al. Easier said than done: keys to successful implementation of the Distress Assessment and Response Tool (DART) program. J Oncol Pract 2016;12:e513-526.

10. Kang JH, Kwon JH, Hui D, et al. Changes in symptom intensity among cancer patients receiving outpatient palliative care. J Pain Symptom Manage 2013;46:652-660.

11. Canadian Medical Association. Policy document PD16-01 - Palliative Care. 2015. Available at: https://www. cma.ca/Assets/assets-library/document/en/advocacy/policy-research/cma-policy-palliative-care-pd16-01-e.pdf. Accessed April 25, 2018.

12. Feldstain A, Lebel $S$, Chasen MR. An interdisciplinary palliative rehabilitation intervention bolstering general self-efficacy to attenuate symptoms of depression in patients living with advanced cancer. Support Care Cancer 2016;24:109-117. 